\title{
Low blood glucose levels in type 2 diabetes: a lot more to come?
}

To the Editor Polish Archives of Internal Medicine (Pol Arch Med Wewn) has recently published a very comprehensive article ${ }^{1}$ about the impact of hypoglycemia on the efficacy of treatment of diabetic patients, with special regard to insulin therapy. Strict glycemic control has been the main goal of antidiabetic therapy in order to decrease the rate of atherotrombotic complications. However, the results of major clinical trials demonstrated that such an approach increased the risk of hypoglycemia. This in turn was shown to increase mortality in patients with diabetes and cardiovascular diseases (CVDs), mainly due to activation of sympathetic nervous system, with subsequent increase in ventricular arrhythmias. Also ischemic complications may participate in hypoglycemia-related increased mortality in a population of patients with diabetes and CVDs. Another clinical state in which hypoglycemia is particularly dangerous and harmful is pregnancy. ${ }^{2}$

Since the publication of the article by Gumprecht et al, ${ }^{1}$ there have been reports of a reduction in the number of hypoglycemia episodes in large Cardiovascular Outcome Trials (CVOT), which focused on cardiovascular (CV) outcomes of new antidiabetic therapies. Moreover, for the first time some of those studies demonstrated reduction in major cardiovascular events. The EMPA-REG OUTCOME study comprised 7020 patients with type 2 diabetes and CVDs, who were randomized to either empagliflozin (10 mg or $25 \mathrm{mg}$ ) or placebo. It was a double-blinded, placebo-controlled study, in which optimal glucose control was the treatment goal in both groups. After 3.1 years of follow-up, there was a significant $14 \%$ reduction in the composite endpoint (nonfatal myocardial infarction, nonfatal stroke, or CV death). Moreover, there was a reduction in CV and total mortality, and a $30 \%-$ to- $35 \%$ reduction in death from heart failure. It was a landmark study, which demonstrated for the first time a reduction in total and CV mortality rates, by adding new oral antidiabetic drug to a standard therapy. Another trial, LEADER, was also a double-blind, placebo-controlled study, in which 9340 patients with type 2 diabetes and high CV risk were randomly assigned to liraglutide or placebo.
The primary composite end-point was the same as in the EMPA-REG OUTCOME study. After 3.8 years of follow-up, the primary outcome was significantly lower in the liraglutide group than in the placebo group, due to fewer CV and all-cause deaths in the interventional group. In both trials, despite better glycemic control in interventional groups, hypoglycemia episodes were not more frequent than in placebo. Interestingly, in the LEADER trial there have been even fewer hypoglycemia incidents in patients on liraglutide than in those on placebo. A reduction in the number of hypoglycemia episodes may be one of the mechanisms accounting for the improvement of $C V$ prognosis, caused by those new antidiabetic drugs. In the light of the data from both trials, the current guidelines were already revised in some countries. ${ }^{3}$

Although insulin therapy is an inevitable treatment in most patients with type 2 diabetes, no trial has demonstrated a reduction in mortality rates resulting from insulin treatment. Recently, preliminary data from the DEVOTE study has shown a significant $40 \%$ reduction in the number of severe hypoglycemia episodes, as well as a $54 \%$ reduction in the number of nocturnal hypoglycemia incidents, in patients treated with a new long-acting insulin analog (degludec) as compared to insulin glargine. Of note, this was associated with a trend (9\%) toward fewer major CV events.

Diabetes is associated with increased platelet aggregation, thrombin generation, and unfavorable fibrin clot properties. Interestingly, not only hyperglycemia but also hypoglycemia is associated with prothrombotic changes and compact fibrin clot formation, which is more resistant to lysis. ${ }^{4}$ Moreover, it has been recently shown that in type 2 diabetes low glucose level $(<4.5-5 \mathrm{mmol} / \mathrm{l})$, which does not fit the criteria for hypoglycemia, is also associated with dense and resistant to lysis fibrin clot, enhanced thrombin generation, and a trend toward increased platelet aggregation. ${ }^{5}$ Those results suggest that active monitoring for low glucose levels preceding hypoglycemia might be worth considering, similarly to screening for prediabetes, especially in the highest-risk patients. ${ }^{6}$ 
To sum up, available new data strengthen the conclusion that besides the treatment of hyperglycemia, avoidance of hypoglycemia should be regarded as an additional therapeutic target in optimal antidiabetic treatment.

Author names and affiliations Grzegorz Gajos, Magdalena Mostowik (GG: Department of Coronary Artery Diseases, Institute of Cardiology, Jagiellonian University Medical College, John Paul II Hospital, Kraków, Poland; MM: Faculty of Medicine, Jagiellonian University Medical College, John Paul II Hospital, Kraków, Poland)

Corresponding author Grzegorz Gajos, MD, PhD, Klinika Choroby Wieńcowej, Instytut Kardiologii, Uniwersytet Jagielloński, Collegium Medicum, Krakowski Szpital Specjalistyczny im. Jana Pawła II, ul. Prądnicka 80, 31-202 Kraków, Poland, phone: +48 1263367 44, e-mail: ggajos@ szpitaljp2.krakow.pl

Conflict of interest The authors declare no conflict of interest.

How to cite Gajos G, Mostowik M. Low blood glucose levels in type 2 diabetes: a lot more to come? Pol Arch Med Wewn. 2016; 126: 1019-1020. doi:10.20452/pamw.3771.

\section{REFERENCES}

1 Gumprecht J, Nabrdalik K. Hypoglycemia in patients with insulin-treated diabetes. Pol Arch Med Wewn. 2016; 126: 870-878.

2 Gutaj P, Zawiejska A, Brązert J, Wender-Ożegowska E. Association between preconceptional treatment with insulin pumps and improved metabolic status in early pregnancy in women with type 1 diabetes. Pol Arch Med Wewn. 2015; 125: 329-336.

3 Canadian Diabetes Association Clinical Practice Guidelines Expert Committee. Pharmacologic Management of Type 2 Diabetes: 2016 Interim Update. Can J Diabetes. 2016; 40: 484-486.

4 Kurdee Z, King R, Ajjan RA. The fibrin network in diabetes: its role in thrombosis risk. Pol Arch Med Wewn. 2014; 124: 617-627

5 Gajos G, Konieczynska M, Zalewski J, Undas A. Low fasting glucose is associated with enhanced thrombin generation and unfavorable fibrin clot properties in type 2 diabetic patients with high cardiovascular risk. Cardiovasc Diabetol. 2015; 14: 44.

6 Sędkowska A, Kowalczyk J, Woźniak A, et al. Role of impaired glucose tolerance in patients with acute myocardial infarction in relation to sex. Pol Arch Med Wewn. 2014; 124: 467-473. 